\title{
The pediatric disease spectrum in emergency departments across Pakistan: data from a pilot surveillance system
}

\author{
Huba Atiq ${ }^{1}$, Emaduddin Siddiqui', Surriya Bano' ${ }^{1}$ Asher Feroze' ${ }^{1}$ Ghazala Kazi ${ }^{1}$, Jabeen Fayyaz ${ }^{1}$, Shivam Gupta ${ }^{2}$, \\ Juanid A Razzak ${ }^{3,5}$, Adnan A Hyder ${ }^{2}$, Asad I Mian ${ }^{1,4^{*}}$
}

\begin{abstract}
Background: There is an increasing number of urgently ill and injured children being seen in emergency departments (ED) of developing countries. The pediatric disease burden in EDs across Pakistan is generally unknown. Our main objective was to determine the spectrum of disease and injury among children seen in EDs in Pakistan through a nationwide ED-based surveillance system.

Methods: Through the Pakistan National Emergency Department Surveillance (Pak-NEDS), data were collected from November 2010 to March 2011 in seven major tertiary care centers representing all provinces of Pakistan. These included five public and two private hospitals, with a collective annual census of over one million ED encounters.

Results: Of 25,052 children registered in Pak-NEDS (10\% of all patients seen): $61 \%$ were male, 13\% under 5 years, while almost $65 \%$ were between 10 to $<16$ years. The majority (90\%) were seen in public hospital EDs. About half the patients were discharged from the EDs, 9\% admitted to hospitals and only 1.3\% died in the EDs. Injury (39\%) was the most common presenting complaint, followed by fever/malaise (19\%) and gastrointestinal symptoms (18\%). Injury was more likely in males vs. females ( $43 \%$ vs. 33\%; $p<0.001)$, with a peak presentation in the $5-12$ year age group (45\%).

Conclusions: Pediatric patients constitute a smaller proportion among general ED users in Pakistan. Injury is the most common presenting complaint for children seen in the ED. These data will help in resource allocation for cost effective pediatric ED service delivery systems. Prospective longer duration surveillance is needed in more representative pediatric EDs across Pakistan.
\end{abstract}

\section{Background}

Each year an increasing number of critically ill children present to emergency departments (EDs) of tertiary-care hospitals of both developing and developed countries [1-3]. The World Health Organization (WHO) conducted a study in 21 hospitals in low-income countries like Bangladesh, Dominican Republic, Ethiopia, and Indonesia, and reported that $90 \%$ of children had severe forms of common infectious diseases, especially pneumonia, diarrhea, sepsis, malaria and meningitis, often complicated with chronic malnutrition [2]. These preventable infectious disease spectrum are the major cause of death and disability

\footnotetext{
* Correspondence: asad.mian@aku.edu

'Department of Emergency Medicine, Aga Khan University, Pakistan
} Full list of author information is available at the end of the article in South Asia, contributing to 3.7 million deaths in children, over $60 \%$ of whom are under 5 years old [5]. How many of these can be readily identified and managed in EDs is unknown. In 2010 there were over 25 million ED visits for children younger than 18 years in the United States; almost $90 \%$ of these visits resulted in release from the ED after treatment [4]. Boys accounted for a slightly larger proportion of ED visits [4]. The mean age of children being seen in the EDs was approximately 7 years; while infants less than one year accounted for $5 \%$ of pediatric ED visits [4]. Injuries and poisoning were common presenting complaints for older children more than 5 years old, while respiratory-related issues were more frequent in the infant age group [4]. Literature from lowincome countries suggests that pediatric emergency 
departments encounter more pediatric patients with medical problems, while a general ED is more likely to treat children with injuries and other musculoskeletal complaints $[1,3,6-8]$. The clinical course of children in a pediatric ED is also unique when compared to children in a general ED; children in pediatric ED tend to be sicker [6-8].

In low- and middle-income countries (LMICs), ill or injured children are often taken to the nearest community-based hospital, irrespective of resources or the ability of the ED to manage the case $[1,3]$. These children may have fatal diseases or arrive too late in the course of the illness, which contributes to rising mortality [3]. According to a recent WHO report, unintentional injuries kill approximately 830,000 children every year, with more than $95 \%$ of those occurring in LMICs $[13,14]$. A global childhood unintentional injuries surveillance done in five major cities of LMICs suggested that falls (50.4\%) and road traffic injuries (16.4\%) were the most common and affect boys more often than girls $(64.7 \%$ vs. $35.3 \%)$ $[13,14]$. Studies on the pediatric ED disease spectrum in developing countries show that common presenting complaints tend to be fever, upper respiratory tract infection and diarrhea [9-12], and common final diagnoses are upper respiratory tract infection (26.7\%), viral syndrome (13.1\%) and gastroenteritis (10.7\%) [9-12]. Therefore, the majority of chief complaints and final diagnoses are infection-related (up to 64\%) [9-12].

Pediatric emergencies can burden limited ED resources, and this is particularly concerning for LMICs. Emergency department related disease spectrum for children across Pakistan is unavailable and thus the case mix of the population is unknown. Knowing the disease burden in terms of frequency of illness or injury in the ED will help plan an effective service delivery system in resource limited settings. The establishment of the Pakistan National Emergency Department Surveillance (Pak-NEDS) was a step towards capturing this information. The primary objective of this study was to determine the burden of pediatric diseases presenting to the EDs of major tertiary care centers across Pakistan. A secondary objective was to investigate if factors such as age, gender, location or type of ED were different among the pediatric diseases being identified through the Pak-NEDS system.

\section{Methods}

\section{Study design and patient enrollment}

The Pakistan National Emergency Departments Surveillance (Pak-NEDS) was a pilot active surveillance done from November 2010 to March 2011. The study sites were seven major EDs in Pakistan: Aga Khan University (AKU) and Jinnah Post-graduate Medical Center in Karachi, Benazir Bhutto Hospital in Rawalpindi, Lady Reading Hospital in Peshawar, Mayo Hospital in Lahore, Sandeman Provincial Hospital in Quetta, and Shifa International Hospital in
Islamabad. All the sites are tertiary care urban centers; AKU and Shifa International Hospital are private hospitals while the rest are public hospitals. AKU was the main coordinating center for the study. All participating EDs are housed in major hospitals in their respective cities, and serve the entire population of those cities. The study participants for Pak-NEDS were males and females of all pediatric age groups presenting to the EDs of participating sites to seek care for any reason, illness or injury. All patients under 16 years were included as cases as it was the age cut off for pediatrics. Ethical approval from all relevant committees was obtained from all participating sites prior to study initiation.

\section{Data collection tool}

A one-page standardized questionnaire was developed based on the ambulatory care survey tool of the Centers for Disease Control and Prevention, USA and previous surveillance work done in Pakistan [11]. The data collection tool was finalized after consultation with the ED heads of all institutions. The tool had questions related to patient demographics like age, gender, ethnicity, presenting complaints, treatment and management provided in the ED and disposition from the ED.

\section{Study procedure}

Data collectors were specifically hired and trained for this study and they worked in three shifts providing 24-hours coverage. Patients were recruited upon registration in the ED. All data was gathered while the patient was in ED, and there was no post ED patient-contact in this study. Data collection was done from the patient or next of kin interviews and ED records. Hard copies of the data collection tool were then sent to the coordinating center at AKUH.

\section{Data entry and analysis}

Data were entered at AKUH using EpiInfo version 3.3.2 and analysis was done using SPSS version 19 . The burden of disease in the pediatric ( $<16$ years) population was analyzed based on the list of presenting complaints and provisional or final diagnoses in the questionnaire. Descriptive data were obtained and reported as mean and median for quantitative variables and proportions for qualitative variables.

\section{Results}

There were 25,052 (9.7\%) patients who were less than 16 years of age in Pak-NEDS; the mean age of pediatric patients enrolled was 10.5 years (SD \pm 4.3 years), with $61 \%$ males (Table 1 ). The majority of patients (65\%) were between 10 to $<16$ years. Patient's gender stratified by age revealed that in all age groups the proportion of males was higher $(60 \%$ for above 5 yrs; $57 \%$ for toddlers and $56 \%$ for infants; $\mathrm{p}<0.008)$. The vast majority of the pediatric 
Table 1 Patient Characteristics of children under 16 years enrolled in Pakistan National Emergency Department Surveillance $(n=25052)$

\begin{tabular}{|c|c|c|}
\hline Variables & $\mathrm{n}$ & $\%$ \\
\hline \multicolumn{3}{|l|}{ Age Group } \\
\hline$<5$ years & 3309 & 13.2 \\
\hline $5-<10$ years & 5357 & 21.4 \\
\hline $10-<16$ years & 16386 & 65.4 \\
\hline \multicolumn{3}{|l|}{ Gender $(n=24715)$} \\
\hline Male & 15102 & 61.1 \\
\hline Female & 9613 & 38.9 \\
\hline \multicolumn{3}{|l|}{ Mode of arrival $(n=23925)$} \\
\hline Ambulance & 744 & 3.1 \\
\hline Non-ambulance & 23181 & 96.9 \\
\hline \multicolumn{3}{|l|}{ Type of hospital } \\
\hline Public hospital & 22303 & 89.0 \\
\hline Private hospital & 2749 & 11.0 \\
\hline \multicolumn{3}{|l|}{ Type of care provider* $(n=23664)$} \\
\hline Paramedic & 10578 & 44.7 \\
\hline House officer/intern & 7860 & 33.2 \\
\hline PG trainee/Resident & 3091 & 13.1 \\
\hline Medical Officer & 16549 & 69.9 \\
\hline Nurse/midwife & 15086 & 63.8 \\
\hline Attending physician & 175 & 0.7 \\
\hline \multicolumn{3}{|l|}{ Disposition $(n=18772)$} \\
\hline Discharged from ED & 8980 & 47.8 \\
\hline Ward admission & 1658 & 8.8 \\
\hline ICU/HDU admission & 125 & 0.7 \\
\hline Observation unit & 2211 & 11.8 \\
\hline Follow-up & 4563 & 24.3 \\
\hline Expired & 250 & 1.3 \\
\hline Others (LAMA/LWBS/Referred out)** & 985 & 5.2 \\
\hline
\end{tabular}

* Multiple response variables

**LAMA, Left against medical advice; LWBS, Left without being seen.

patients in Pak-NEDS presented at public hospitals (89\%). Almost $97 \%$ were brought to the ED by non-ambulance means, while a much smaller proportion $(3.1 \%)$ were brought by ambulance services. The median time to ED presentation was 170 minutes [Inter-quartile range (IQR) 40-721 minutes]. About $70 \%$ were evaluated by a medical officer, $63.8 \%$ by a nurse or midwife, and a small proportion $(0.7 \%)$ was evaluated by an attending physician (Table 1 ).

Information pertaining to patient status at time of arrival was lacking in $12 \%$ of cases; $0.5 \%$ were reported dead at the time of arrival to the ED. Data on ED disposition was available for $74 \%$ of patients; almost half were discharged (48\%), $8.9 \%$ were admitted and $1.3 \%$ of cases were reported to have died while in the ED (Table 1). This represents a mortality rate of 1300 per 100,000 pediatric patients seen in the ED.

The "presenting complaint" variable was used to analyze the disease spectrum among the pediatric age group by the ED physicians (Table 2). Of the 22,047 cases, injury
Table 2 Top 10 presenting complaints for pediatric patients in Pakistan National Emergency Department Surveillance $(N=22047)$

\begin{tabular}{|c|c|c|}
\hline Presenting Complaints for Patients $<16$ years & $\mathbf{N}$ & $\%$ \\
\hline Injury & 8680 & 39.4 \\
\hline Constitutional* & 4160 & 18.9 \\
\hline Gastrointestinal & 4025 & 18.3 \\
\hline Respiratory & 3018 & 13.7 \\
\hline Neurological & 2825 & 12.8 \\
\hline Cardiovascular & 1391 & 6.3 \\
\hline Musculoskeletal & 1332 & 6 \\
\hline Eye \& Otolaryngology & 500 & 2.3 \\
\hline Genitourinary & 457 & 2.1 \\
\hline Others $^{+}$ & 679 & 3.1 \\
\hline
\end{tabular}

*Fever, lethargy, lassitude, weakness, loss of appetite, etc.

${ }^{+}$Skin, Psychiatric

was the most frequent complaint reported at the time of arrival in ED (39.4\%), followed by constitutional symptoms such as fever and malaise (18.9\%) and gastrointestinal symptoms $(18.3 \%)$. The presenting complaints were further stratified by gender, age, province and the type of hospital (Table 3). Females had a higher percentage of gastrointestinal, respiratory and neurological presenting complaints as compared to males, while males had comparatively higher percentage related to injury (43.4\% as compared to $33 \%$ in female; $\mathrm{p}<0.001$ ). Presenting complaints relating to constitutional (fever, malaise, etc.), cardiovascular and musculoskeletal symptoms were similar among both genders. When stratified by age, there were fewer cases of neurological and musculoskeletal symptoms among children under 5 (4.6\% and $5.8 \%$ respectively) as compared to older age groups. Similarly, for cardiovascular symptoms, there were fewer children under 5 years with these presenting complaints (7.4\%) versus the other age groups (Table 3). Children under 5 years were more likely to present with injury and constitutional-related presenting complaints, than older age groups $(\mathrm{p}<0.001)$. Of the children with injury as a chief presenting complaint, $42 \%$ were seen at a public hospital ED, whereas children with gastrointestinal, respiratory, and neurological emergencies reported mostly to private hospitals $(\mathrm{p}<0.001)$ (Table 3 ). A significant difference was observed in case mortality rates in the EDs, between public and private facilities $(25$ per 1000 children in a private ED vs. 12 per 1000 children in a public ED; $\mathrm{p}<0.001$ ).

\section{Discussion}

This study is the first of its kind to describe the pediatric disease spectrum in EDs across Pakistan. The disease frequency revealed in this study was from general EDs across the country and thus might not be wholly representative of the actual disease spectrum as observed in dedicated pediatric EDs [5,10-12]. What is 
Table 3 Presenting complaints stratified by patient characteristics for pediatric patients under 16 years of age in Pakistan National Emergency Department Surveillance $(n=22047)$

\begin{tabular}{ccccccccc}
\hline & \multicolumn{7}{c}{ Disease Spectrum n(\%) } \\
\cline { 2 - 9 } & Constitutional & Cardiovascular & Gastrointestinal & Respiratory & Neurological & Musculoskeletal & Injury & Total \\
\hline Gender & 4123 & 1358 & 3965 & 2997 & 2815 & 1315 & 8573 & $\mathbf{2 1 7 7 7}$ \\
\hline Male & $2561(19.1)$ & $754(5.6)$ & $2203(16.4)$ & $1657(12.4)$ & $1492(11.1)$ & $754(5.6)$ & $5810(43.4)$ & $\mathbf{1 3 3 9 6}$ \\
Female & $1562(18.6)$ & $604(7.2)$ & $1762(21)$ & $1340(16)$ & $1323(15.8)$ & $561(6.7)$ & $2763(33)$ & $\mathbf{8 3 8 1}$ \\
P-value & NS & $<0.001$ & $<0.001$ & $<0.001$ & $<0.001$ & 0.001 & $<0.001$ & - \\
\hline Age Group & 4160 & 1391 & 4025 & 3018 & 2825 & 1332 & 8680 & $\mathbf{2 2 0 4 7}$ \\
\hline$<5$ years & $729(24.9)$ & $218(7.4)$ & $566(19.3)$ & $390(13.3)$ & $135(4.6)$ & $170(5.8)$ & $1126(38.4)$ & $\mathbf{2 9 3 1}$ \\
5-9 years & $960(20.0)$ & $274(5.7)$ & $715(14.9)$ & $591(12.3)$ & $395(8.2)$ & $248(5.2)$ & $2330(48.6)$ & $\mathbf{4 7 9 0}$ \\
10-15 years & $2471(17.2)$ & $899(6.3)$ & $2744(19.2)$ & $2037(14.2)$ & $2295(16.0)$ & $914(6.4)$ & $5224(36.5)$ & $\mathbf{1 4 3 2 6}$ \\
P-value & $<0.001$ & 0.010 & $<0.001$ & $<0.01$ & $<0.001$ & $<0.01$ & $<0.001$ & - \\
\hline Type of hospital & 4160 & 1391 & 4025 & 3018 & 2825 & 1332 & 8680 & $\mathbf{2 2 0 4 7}$ \\
\hline Public hospital & $3568(18.3)$ & $1211(6.2)$ & $3358(17.2)$ & $2453(12.6)$ & $2185(11.2)$ & $1220(6.3)$ & $8168(41.9)$ & $\mathbf{1 9 5 0 5}$ \\
Private hospital & $592(23.3)$ & $180(7.1)$ & $667(26.2)$ & $565(22.2)$ & $640(25.2)$ & $112(4.4)$ & $512(20.1)$ & $\mathbf{2 5 4 2}$ \\
P-value & $<0.001$ & 0.089 & $<0.001$ & $<0.001$ & $<0.001$ & $<0.001$ & $<0.001$ & - \\
\hline
\end{tabular}

noteworthy in this study is that only $10 \%$ of patients enrolled through Pak-NEDS belonged to the pediatric age group. One reason for this could be that children might be taken to EDs that deal with children rather than being brought to general EDs. The literature also suggests that a dedicated pediatric ED encounters more patients with medical problems while a general ED is more likely to treat children with injuries and other musculoskeletal complaints $[1,6,7]$. The clinical course of children in a pediatric ED is also unique when compared to children in a general ED; for example, children in a pediatric ED tend to be sicker at admission [6-10]. Furthermore, the burden of chronic illness in LMICs is higher in a pediatric ED due to referral from the primary physicians or sub-specialists $[15,16]$. Pediatric EDs are typically in children's hospitals versus pediatric sections in general EDs. How this impacts pediatric emergency care in resource-limited settings is not entirely clear, as there are likely to be even fewer dedicated children's hospitals with pediatric emergencies in such settings [7].

Injuries accounted for almost half of the presenting complaints to the EDs $[8,17]$ which, considering the high morbidity and mortality related to trauma, poses a significant burden on hospitals [13,14,17-20]. Although this study was different from the previous ED-based surveillance study focused specifically on childhood unintentional injuries in LMICs including Pakistan [17-20], both studies revealed that boys were more likely to be injured. Most injuries were minor; however, the predominance of head, face and limb injuries might lead to significant future morbidity and disability. Furthermore, the possibility that some trauma was non-accidental or intentional cannot be overlooked.

Pediatric infection-related ailments were also relatively frequent presenting complaints. This is also consistent with literature from Pakistan and other LMICs as presenting complaints of constitutional symptoms (like fever, malaise, lethargy), gastrointestinal (diarrhea, vomiting), acute respiratory and neurological symptom could all indicate infectious etiology [21-23]. In fact, Pakistan is one of five countries with the highest under five mortality due to infectious disease [2-5,24].

It is important to keep in mind that Pakistan has few established EDs that are led by formally trained emergency medicine staff [25-29]. EDs (commonly called casualty rooms) are primarily run by junior medical officers with limited expertise in delivering pediatric care $[25,26,29]$. These medical officers are only capable of handling the minor illnesses while more complicated and serious cases are being immediately referred to specialist pediatric residents on-call or to other pediatric hospital $[26,27]$. The EDs at basic health units or the primary health centers in rural or peri-urban vicinities are run by paramedics, nurses or health workers with no or minimal training in pediatric care $[26,27]$. Consistent with this, most patients in this study were either seen by a medical officer or paramedic staff, with less than $1 \%$ of the children being seen by an ED or pediatric consultant $[26,27]$.

In addition to this, the structured pediatric teachings at undergraduate level started in Pakistan in the late 1990's [25-27]. Though there are post-graduate examinations in general pediatrics, pediatric emergency training and examinations are yet to start in the country. This highlights the need for improved training and development of pediatric EDs across the country utilizing a uniform evidence-based medicine approach, particularly for the commonly encountered pediatric ailments in Pakistan. 
Ambulance service utilization was found to be negligible in this study, highlighting the need for a well-defined pediatric ambulance service across Pakistan. Reasons for preferential utilization of private transport may include the lack of a well-coordinated nationwide emergency medical service, and lack of awareness regarding usage of ambulances [30-32].

This study has several limitations. The missing data for important variables can be explained by the fact that this was a nationwide surveillance dependent on manpower availability. A centralized computer system keeping track of data collected was also lacking. The diverse and unpredictable nature of ED work flow might have contributed to inefficient data collection. Emergencies like bomb blast across the country result in chaotic situation in EDs which is likely to contribute to problems in data collection. Another limitation was the collection of multiple variable responses. By allowing the patient or family member to report up to three responses, a chief complaint was sometimes difficult to determine in the post hoc analysis. However, for the major analyses, it was a very small percentage of patients that reported any overlap in categories, such as injury and infection. In spite of the above limitations, this study presents a comprehensive data set on almost 25,000 pediatric patient encounters in a few representative EDs across Pakistan.

\section{Conclusion}

Almost half of pediatric presentations to EDs across Pakistan are injury-related. Although alarming, this provides an opportunity to reinforce pediatric trauma management services in EDs, along with screening for potential victims of child abuse. Infection-related complaints are also frequently encountered in pediatric ED visits and this can be a potential opportunity to enhance care by the development and uniform implementation of pediatric emergency medicine guidelines for commonly encountered infectious disease-related problems.

A logical next step would be a surveillance study that is of longer duration, utilizes more comprehensive data collection and more rigorous and robust computer-mediated data entry, and enrolls patients in many more representative EDs across Pakistan, including those of children's hospitals.

\section{Funding statement}

The Pak-NEDS study was supported through the "Johns Hopkins International Collaborative Trauma and Injury Research Training Program" [Grant No. D43TW007292] by Fogarty International Center of the United States National Institutes of Health. The content is solely the responsibility of the authors and does not represent the views of Fogarty or NIH.

\section{Competing interests}

The authors declare that they have no competing interests.

\section{Authors' contributions}

HA, EUS, SB, AF, GK, JF developed the initial draft and analysis plan. HA, EUS, $A F$ and AIM were involved in data analysis and interpretation. JAR, AAH and AIM provided critical review of the draft. JAR and AAH were involved in the overall design and implementation of Pak-NEDS. All authors approved of the final draft.

\section{Acknowledgements}

We are grateful to Ms. Bobbi Nicotera for providing language edits of the draft. We also acknowledge the data collection team and local collaborators for their support during data collection process.

This article has been published as part of BMC Emergency Medicine Volume 15 Supplement 2, 2015: Articles from the Pakistan National Emergency Departments Surveillance Study (Pak-NEDS). The full contents of the supplement are available online at http://www.biomedcentral.com/ bmcemergmed/supplements/15/S2. Publication of this supplement was funded by the Johns Hopkins School of Public Health.

\section{Authors' details}

${ }^{1}$ Department of Emergency Medicine, Aga Khan University, Pakistan. ${ }^{2} J o h n s$ Hopkins International Injury Research Unit, Department of International Health, Johns Hopkins Bloomberg School of Public Health, Baltimore Maryland, USA. ${ }^{3}$ Department of Emergency Medicine, Johns Hopkins School of Medicine, Baltimore, Maryland, USA. ${ }^{4}$ Department of Paediatrics and Child Health, Aga Khan University, Pakistan. ${ }^{5}$ The author was affiliated with the Department of Emergency Medicine, Aga Khan University, Karachi, Pakistan at the time when study was conducted.

\section{Published: 11 December 2015}

\section{References}

1. Goh AY, Chan TL, Abdel-Latiff ME: Paediatric utilization of a general emergency department in a developing country. Acta Paediatrica 2003, 92(8):965-969.

2. Nolan $T$, Angos P, Cunha AJLA, Muhe L, Qazi S, Simoes EAF, Tamburlini G, Weber M, Pierce NF: Quality of hospital care for seriously ill children in less-developed countries. The Lancet 2001, 357(9250):106-110.

3. Singhi S, Jain V, Gupta G: Pediatric emergencies at a tertiary care hospital in India. Journal of tropical pediatrics 2003, 49(4):207-211.

4. Wier LM, Yu H, et al: Overview of Children in the Emergency Department. 2010.

5. Zaidi AKM, Awasthi S, deSilva HJ: Burden of infectious diseases in South Asia. Bmj 2004, 328(7443):811-815.

6. Bourgeois FT, Shannon MW: Emergency care for children in pediatric and general emergency departments. Pediatric emergency care 2007, 23(2):94-102.

7. Molyneux E: Emergency care for children in resource-constrained countries. Transactions of the Royal Society of Tropical Medicine and Hygiene 2009, 103(1):11-15

8. Nelson DS, Walsh K, Fleisher GR: Spectrum and frequency of pediatric illness presenting to a general community hospital emergency department. Pediatrics 1992, 90(1):5-10.

9. Massin MM, Montesanti J, Gerard P, Lepage P: Spectrum and frequency of illness presenting to a pediatric emergency department. Acta clinica Belgica 2006, 61(4):161-165.

10. Mintegi S, Shavit I, Benito J, group R: Pediatric emergency care in Europe: a descriptive survey of 53 tertiary medical centers. Pediatric emergency care 2008, 24(6):359-363.

11. National Center for Health S: National Hospital Ambulatory Medical Care Survey: Emergency department summary. US Department of Health and Human Services, Public Health Service, Centers for Disease Control and Prevention, National Center for Health Statistics 1999.

12. Bazaraa HM, El Houchi S, Rady HI: Profile of patients visiting the pediatric emergency service in an Egyptian university hospital. Pediatric emergency care 2012, 28(2):148-152.

13. Hyder AA, Sugerman DE, et al: Global childhood unintentional injury surveillance in four cities in developing countries: a pilot study. Bulletin of the World Health Organization 2009, 87(5):345-352. 
14. He S, Lunnen JC, et al: Global childhood unintentional injury study: Multisite surveillance data. American journal of public health 2014, 104(3):e79-e84

15. Massin MM, Montesanti J, Gerard P, Lepage P: Children with chronic conditions in a paediatric emergency department. Acta Paediatrica 2006, 95(2):208-13.

16. Reynolds S, Desguin B, Uyeda A, Davis AT: Children with chronic conditions in a pediatric emergency department. Pediatric emergency care 1996, 12(3):166-168.

17. Hyder AA, Razzak JA: The challenges of injuries and trauma in Pakistan: An opportunity for concerted action. Public health 2013, 127(8):699-703.

18. Fatmi Z, Kazi A, Hadden WC, Bhutta ZA, Razzak JA, Pappas G: Incidence and pattern of unintentional injuries and resulting disability among children under 5 years of age: results of the National Health Survey of Pakistan. Paediatric and perinatal epidemiology 2009, 23(3):229-238.

19. Nasrullah M, Muazzam S: Risk differences between children and adults in road traffic injuries: a descriptive study from a tertiary-care hospital in a low-income country. European Journal of Emergency Medicine 2012, 19(3):167-170.

20. Razzak JA, Khan UR, Zia N, Azam I: A child an hour: burden of injury deaths among children under 5 in Pakistan. Archives of disease in childhood 2013, 98(11):867-871.

21. Bhutta ZA, Hafeez A, Rizvi A, Ali N, Khan A, Ahmad F, Bhutta S, Hazir T, Zaidi A, Jafarey SN: Reproductive, maternal, newborn, and child health in Pakistan: challenges and opportunities. The Lancet 2013, 381(9884):2207-2218.

22. Owais A, Tikmani SS, Sultana S, Zaman U, Ahmed I, Allana S, Zaidi AKM: Incidence of pneumonia, bacteremia, and invasive pneumococcal disease in Pakistani children. Tropical Medicine \& International Health 2010, 15(9):1029-1036.

23. Walker CLF, Rudan I, Liu L, Nair H, Theodoratou E, Bhutta ZA, O'Brien KL, Campbell H, Black RE: Global burden of childhood pneumonia and diarrhoea. The Lancet 2013, 381(9875):1405-1416.

24. Gentile A, Bhutta Z, Bravo L, Samy AG, Garcia RDJ, Hoosen A, Islam T, Karimi A, Salem M, Simasathien S: Pediatric disease burden and vaccination recommendations: understanding local differences. International Journal of Infectious Diseases 2010, 14(8):e649-e658.

25. Mehdi IJ: Emergency medicine in Pakistan. Annals of emergency medicine 1996, 27(1):84-88.

26. Raftery KA: Emergency medicine in southern Pakistan. Annals of emergency medicine 1996, 27(1):79-83.

27. Razzak JA, Ahmed A, Saleem AF, Nasrullah M: Perceived need for emergency medicine training in Pakistan: a survey of medical education leadership. Emergency Medicine Australasia 2009, 21(2):143-146.

28. Razzak JA, Kellermann AL: Emergency medical care in developing countries: is it worthwhile? Bulletin of the World Health Organization 2002 80(11):900-905.

29. Shahid M, Razzak J, Jamali S, Ali SS, Ayubi TK: Initial diagnostic categories of patients presenting to four major emergency departments in Karachi. Journal of the College of Physicians and Surgeons of Pakistan 2006, 16(10):680.

30. Baqir M, Ejaz K: Role of pre-hospital care and ambulance services in Karachi. JPMA-Journal of the Pakistan Medical Association 2011, 61(12):1167.

31. Chandran A, Ejaz K, Karani R, Baqir M, Razzak J, Hyder AA: Insights on the effects of patient perceptions and awareness on ambulance usage in Karachi, Pakistan. Emergency Medicine Journal 2014, 31:990-993.

32. Naseer R, Tahir N, Akbar AH: Role of rescue 1122 in the development of safer communities in Pakistan. Injury Prevention 2012, 18(Suppl 1):A89-A89.

doi:10.1186/1471-227X-15-S2-S11

Cite this article as: Atiq et al:: The pediatric disease spectrum in emergency departments across Pakistan: data from a pilot surveillance system. BMC Emergency Medicine 2015 15(Suppl 2):S11.

\section{Submit your next manuscript to BioMed Central and take full advantage of:}

- Convenient online submission

- Thorough peer review

- No space constraints or color figure charges

- Immediate publication on acceptance

- Inclusion in PubMed, CAS, Scopus and Google Scholar

- Research which is freely available for redistribution

Submit your manuscript at www.biomedcentral.com/submit 